\title{
Surface enhanced Raman scattering of new acridine based fluorophore adsorbed on silver electrode
}

\author{
Elena V. Solovyeva, Liubov A. Myund, Anna S. Denisova* \\ Chemistry Institute, Saint-Petersburg State University, Universitetsky pr. 26, Stary Peterhof, Saint-Petersburg 198504, Russian Federation
}

\section{H I G H L I G H T S}

- SERS spectra of BHIA arise despite the bulky substituents near of acridine nitrogen.

- BHIA and BHIA-H+ interact with silver electrode surface via the $\pi$ aromatic system.

- Sensoric fragment of adsorbed BHIA is available for both protonation and complexation.
G R A P H I C A L A B S T R A C T

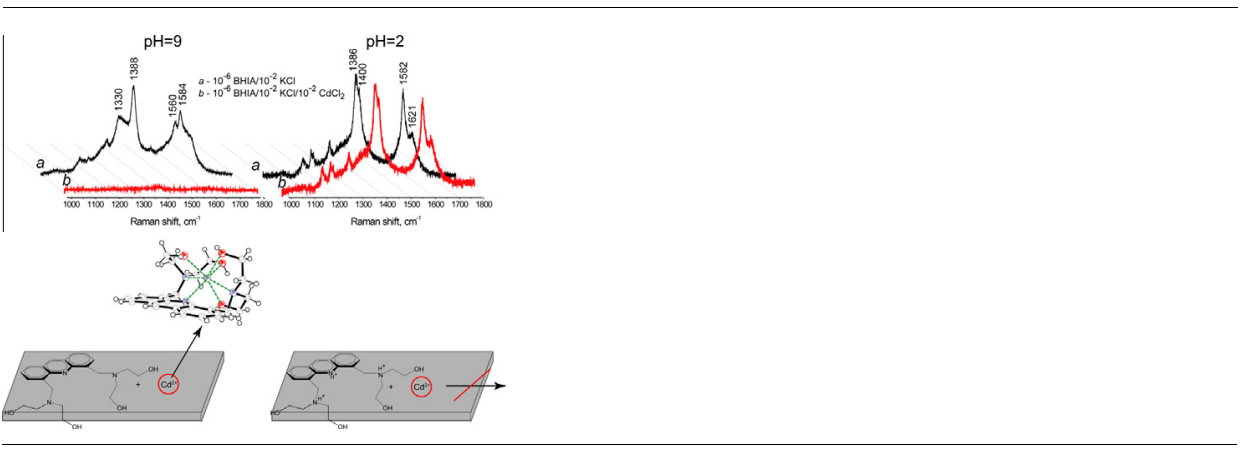

\section{A R T I C L E I N F O}

\section{Article history:}

Received 29 October 2014

Received in revised form 18 April 2015

Accepted 21 April 2015

Available online 2 May 2015

\section{Keywords:}

Surface-enhanced Raman spectroscopy

Fluorophore

Acridine derivatives

Adsorption

\begin{abstract}
A B S T R A C T
4,5-Bis(N,N-di(2-hydroxyethyl)iminomethyl)acridine (BHIA) is a new acridine based fluoroionophore and a highly-selective sensor for cadmium ion. The direct interaction of the aromatic nitrogen atom with a surface is impossible since there are bulky substituents in the 4,5-positions of the acridine fragment. Nevertheless BHIA molecule shows a reliable SERS spectrum while adsorbed on a silver electrode. The analysis of SERS spectra pH dependence reveals that BHIA species adsorbed on a surface can exist in both non-protonated and protonated forms. The adsorption of BHIA from alkaline solution is accompanied by carbonaceous species formation at the surface. The intensity of such "carbon bands" turned out to be related with the supporting electrolyte $(\mathrm{KCl})$ concentration. Upon lowering the electrode potential the SERS spectra of BHIA do not undergo changes but the intensity of bands decreases. This indicates that the adsorption mechanism on the silver surface is realized via aromatic system of acridine fragment. In case of such an adsorption mechanism the chelate fragment of the BHIA molecule is capable of interaction with the solution components. Addition of $\mathrm{Cd}^{2+}$ ions to a system containing BHIA adsorbed on a silver electrode in equilibrium with the solution leads to the formation of $\mathrm{BHIA} / \mathrm{Cd}^{2+}$ complex which desorption causes the loss of SERS signal.
\end{abstract}

(c) 2015 Elsevier B.V. All rights reserved.

\section{Introduction}

Surface-enhanced Raman spectroscopy (SERS) is a powerful tool for surface chemistry and physics investigation. Currently the SERS method is used for both the study of surface itself [1-4] and the

\footnotetext{
* Corresponding author. Tel.: +7 812428 4062; fax: +7 8123636722.

E-mail address: anna@raman.org.ru (A.S. Denisova).
}

investigation of the adsorbed molecules [5-9]. In terms of adsorption chemistry SERS is particularly valuable because there are no alternative locally sensitive methods for the investigation the state of the adsorbate. The study of adsorbates by SERS spectroscopy provides information about their transformations on the surface [10-13]. The SERS technique applied to surface-solution boundary can give information about the reorientation of adsorbates [14-17] and their interaction with the solution components [18-21]. 
The interaction between molecules and a surface is necessary for the appearance of SERS spectrum. If a molecule has several possible fragments for adsorption the problem arises to determine through which of them it interacts with the surface. The understanding of the adsorption manner is very important both for the insight into the SERS nature and for practical purposes for example for the targeted development of functional self-assembled monolayers.

The aromatic heterocyclic compounds are capable to be adsorbed via the lone electron pair of a heteroatom or via the conjugated aromatic system. The adsorption of azaheterocycle molecules such as pyridine, phenanthroline, acridine, etc. on silver surface have been studied by the SERS method in detail but clear understanding of their adsorption manner is not achieved yet. The hypothetical adsorption model with the key role of lone electron pair of the nitrogen atom in the heterocycle was considered for pyridine [22-25] and phenanthroline [26-28]. In the case of acridine the type of SERS spectra dependence on electrode potential [29] and stability of the SERS spectrum of protonated form over time [29-31] suggest that the conjugate aromatic system takes an important part in the adsorption process along with the lone electron pair. This fact stimulates an interest to investigation of the adsorption of acridine derivatives with bulky substituents near the nitrogen atom. For such derivatives, the direct interaction of the molecule with a surface via the lone electron pair of aromatic nitrogen becomes hardly possible. Some newly synthesized acridine derivatives with substituents in the 4,5-positions are the most attractive as highly selective fluorophores [32-36]. The study of such substances immobilized on the surface (in particular the information on the availability of their sensor fragment for solution components) is very important with respect to analytical applications (for the detection limit lowering due to surface enhancement effect) as well as to clearer understanding of the adsorption mechanism. Thus, the results of this work may be of significance not only for the development of the SERS method but for surface enhanced infrared absorption (SEIRA) and surface enhanced fluorescence also.

We have studied here, for the first time, the SERS spectra of acridine derivative 4,5-bis(N,N-di(2-hydroxyethyl)iminomethyl)acridine (BHIA, see Fig. 1) which is chemosensor for $\mathrm{Cd}^{2+}[34]$.

\section{Experimental}

BHIA $\left(\mathrm{C}_{23} \mathrm{H}_{31} \mathrm{~N}_{3} \mathrm{O}_{4}\right)$ (99\%) was purchased from Synthon-Lab (Russia)

The SERS spectra were recorded using a DFS-52 (LOMO, USSR) spectrometer. The $488 \mathrm{~nm}$ line of $\mathrm{Ar}^{+}$laser LGN-503 was used to excite the spectra. The spectra were recorded by a FEU-79 photomultiplier in the photon counting mode. The accuracy of the wave<smiles>OCCN(CCO)Cc1cccc2cc3cccc(CN(CCO)CCO)c3nc12</smiles>

Fig. 1. The structure of BHIA. number reproduction was $0.1 \mathrm{~cm}^{-1}$. The accumulation time per spectral point is $0.1 \mathrm{~s}$, the total accumulation time for spectrum is about $3 \mathrm{~min}$. The laser power was $200 \mathrm{~mW}$ and the diameter of spot at the sample is approximately $2 \mathrm{~mm}$ in all measurements. The line from the spirit of diffraction grating presents in the spectra at $1200 \mathrm{~cm}^{-1}$.

The SERS study of BHIA was performed with a silver electrode as active substrate in the electrochemical cell with a saturated $\mathrm{Ag} \mid \mathrm{AgCl}$ reference electrode and platinum foil as counter electrode. Before measurements, the silver electrode was etched with strong nitric acid and carefully rinsed with distilled water. In all cases BHIA was adsorbed by electroadsorption at potential of $-200 \mathrm{mV}$ from the $10^{-6} \mathrm{M}$ aqueous BHIA solution containing $10^{-2} \mathrm{M} \mathrm{KCl}$ as a supporting electrolyte.

The solutions with different $\mathrm{pH}$ values were prepared by addition of suitable volumes of $\mathrm{HCl}$ or $\mathrm{KOH}$ to the BHIA solution. The $\mathrm{pH}$ was measured by the pH-glass electrode ESL 43-07, Izmeritel, Gomel, Belarus.

\section{Results and discussion}

The BHIA SERS spectra recorded at different $\mathrm{pH}$ values are presented in Fig. 2. One can see that the BHIA molecule shows a good SERS spectrum when adsorbed on the silver electrode. The bulky substituents are seen not to interfere with the interaction of BHIA with surface sufficient for the spectrum to arise. To insight in the origin of enhancement of the Raman spectrum of BHIA its UV-visible absorption spectra may be helpful. The absorption spectra of solution of BHIA 4,5-bis(N,N-di(2-hydroxyethyl)iminomethyl)acridine (BHIA) and its complex with $\mathrm{Cd}$ were studied

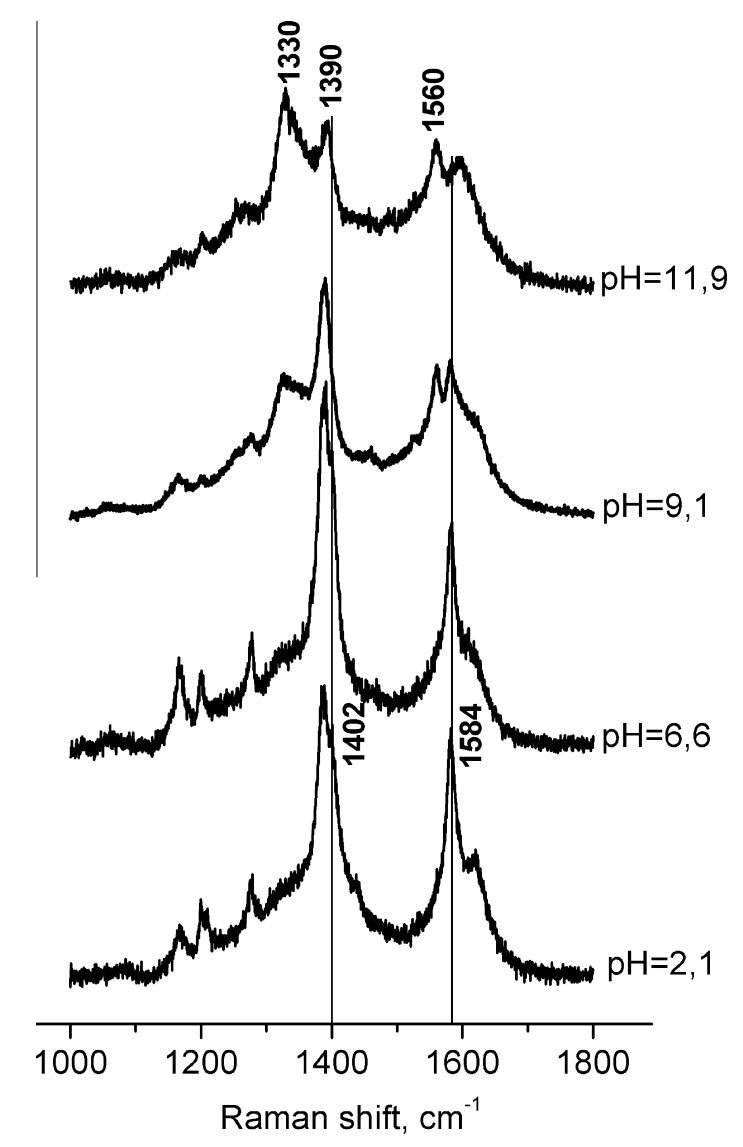

Fig. 2. SERS spectra of BHIA adsorbed on silver electrode at different pH values. All spectra are normalized to integral intensity. 
in [34]. The most significant difference among these spectra is observed for the band at $253 \mathrm{~nm}$. The bands with a maximum wavelength are $390-395 \mathrm{~nm}$ for the BHIA and $420-425 \mathrm{~nm}$ for the BHIA $/ \mathrm{Cd}^{2+}$ complex. This fact suggests that at the excitation of Raman spectra by $488 \mathrm{~nm}$ line the condition of resonance with electronic transitions of BHIA molecule is not realized. On the other hand the $488 \mathrm{~nm}$ line lies within the region of electromagnetic resonance with silver nanoparticles. In order to get more information about the contributions of the electromagnetic resonance and the electronic structure of molecule to the enhancement of BHIA Raman spectrum, the investigation of SERS spectra at different excitation wavelengths may be carried out.

The SERS spectrum of BHIA resembles the one of acridine [29]. As in the normal Raman, all main spectral components observed are similar for both substances. The investigation of BHIA IR and normal Raman spectra and their assignment based on the DFT calculations of optimized geometry and normal modes of wavenumbers were carefully performed elsewhere [37]. According to the assignment of the vibrational spectrum of BHIA it may be considered that all bands in the presented SERS spectrum relate to the ring in-plane vibrations of aromatic fragment. For all bands the shifts of their positions compared to the normal Raman spectrum are non-significant, and lay within the range of $6-9 \mathrm{~cm}^{-1}$.

As well as the acridine molecule [29], BHIA may exist both as non-protonated and protonated species adsorbed on the surface. The most significant differences for both species are observed for the shoulder at $1402 \mathrm{~cm}^{-1}$ and the band at $1584 \mathrm{~cm}^{-1}$. The $\mathrm{pH}$ value increase leads to the disappearance of the first frequency and the shift of the second one to $1560 \mathrm{~cm}^{-1}$. According to the assignment of BHIA Raman spectrum the band at $1402 \mathrm{~cm}^{-1}$ corresponds to the normal vibration arising mainly from the ring-inplane displacements of atoms of the aromatic fragment and from wagging vibration of $\mathrm{CH}_{2}$ groups of outlying hydroxyethylic aliphatic fragments; the band at $1584 \mathrm{~cm}^{-1}$ is related to the ring-in-plane vibration of acridine fragment of molecule.

As one can see from Fig. 2 together with the change of the spectrum profile the fall of intensity occurs at alkaline $\mathrm{pH}$. Probably, active properties of surface become worse in alkaline solution due to the partial formation of silver oxide.

There are three centers of protonation in the BHIA molecule: the aromatic nitrogen atom (pKa for acridine $~ 5.6$ [38]) and two benzylic nitrogen atoms (pKa 7.38 [34]). The protonation of amine nitrogens appears in the fluorescence spectra of BHIA because the quantum yield strongly depends on photoinduced electron transfer (PET) from amine nitrogens to excited acridine. The PET mechanism is suppressed when benzylic nitrogens are converted to the corresponding ammonium ions [34]. Since the bands corresponding to the ring in plane vibrations present in the studied region of BHIA SERS spectrum mainly the spectrum evolution is caused by the protonation of aromatic nitrogen atom. Furthermore the similar changes were observed in the SERS spectra of acidic/basic forms of acridine. Thus there is the principal difference between fluorescence and SERS spectra concerning their sensitivity to the protonation of BHIA: fluorescent signals reflect the protonation of the amine nitrogens while the SERS signals reflect the protonation of aromatic nitrogen atom.

Broad featureless scattering signals near 1330 and $1588 \mathrm{~cm}^{-1}$ in the spectra particularly noticeable at alkaline $\mathrm{pH}$ apparently are not relevant to the BHIA molecule. The presence of these components in the SERS spectra is not uncommon and is peculiar to various substances. Currently these bands are correlated with a carbonaceous species on the metal surface as evidenced by numerous studies [39-41]. Despite the presence in spectra of features from carbonaceous species probably occurs due to using the relatively high laser power it is interesting to note that the process of carbon formation on the surface has a "chemical cause". In addition to the dependence on the $\mathrm{pH}$ of solution this also follows from the influence of the supporting electrolyte concentration on the intensity of the bands centered at 1330 and $1588 \mathrm{~cm}^{-1}$.

As can be seen from Fig. 3, the intensity of the bands from BHIA does not change with the increasing $\mathrm{KCl}$ concentration, while the 1330 and $1588 \mathrm{~cm}^{-1}$ components intensity diminishes greatly. This phenomenon can be interpreted from the following point of view: chloride anions have a greater affinity to the surface than the carbonaceous species and therefore displace the latter from the surface. The higher the chloride anions concentration the more carbon is displaced from the surface. Two factors can be responsible for the significant intensity of carbon bands in alkaline solution: firstly the more intense graphitization and secondly the content of chloride anions in the double electric layer at $\mathrm{pH}=12$ is comparable with the $\mathrm{OH}^{-}$concentration. The results presented in Fig. 3 can be of practical importance as the supporting electrolyte concentration increase can be used as in situ method for cleaning the background of SERS spectrum from undesirable carbon components. Importantly, this method does not require the working cell disassembling.

The dependence of the BHIA SERS spectra on the electrode potential provides information about the alteration of the adsorbed molecule state upon the variation of the surface potential. In the acidic solution, the spectrum intensity is decreased with the shift of electrode potential to the negative range whereas the spectral profile remains unchanged (see Fig. 4). The changes occurring with the spectrum are almost the same for the alkaline solution as well. This indicates that while the electrode potential shifts, the protonated and non-protonated forms of BHIA are desorbed from the surface instead of being reoriented. It should be emphasized that the behavior of non-protonated form of acridine over the change of the electrode potential is quite different. According to [29], the intensity of the acridine spectrum components remains nearly unchanged over the shift of potential to more negative values. In contrast to the acridine desorption, the desorption of non-protonated BHIA molecules occurs because their direct interaction with the surface via the lone electron pair of nitrogen atom becomes impossible. The similar influence of the electrode potential on the SERS spectra of protonated and non-protonated forms of BHIA proves that the both forms adsorb on the surface in the same way. It may be supposed that BHIA molecules interact with the surface via the $\pi$-system of aromatic rings. Thus, the desorption of BHIA is caused by the repulsion of the $\pi$-system from the negative-charged surface.

The investigation of the BHIA SERS spectra in the systems containing complexing cations is of a great interest in view of its sensory properties. Non-protonated and protonated forms of BHIA demonstrate different responses to $\mathrm{Cd}^{2+}$ addition to solution.

As can be seen from Fig. 5, the SERS spectrum of the non-protonated form disappears when $\mathrm{Cd}^{2+}$ is added to the solution whereas the spectrum of the protonated form remains unchanged. Adsorbed BHIA molecules interact in alkali media with cadmium cations forming BHIA $/ \mathrm{Cd}^{2+}$ complex. In accordance with the X-ray single crystal structural analysis data, the conformation of the BHIA molecule changes dramatically upon complexation compared to the free ligand [37]. Apparently, this results in the inability of the BHIA $/ \mathrm{Cd}^{2+}$ complex to remain on the surface. The desorption of BHIA $/ \mathrm{Cd}^{2+}$ complex from the surface to the solution causes the loss of SERS signal (Fig. 6a). Since the protonation constants of all BHIA nitrogen atoms are higher than the BHIA $/ \mathrm{Cd}^{2+}$ complexation constant which was found to be $3.66 \times 10^{4} \mathrm{~L} \mathrm{~mol}^{-1}$ [34] there is no interaction of BHIA molecules with $\mathrm{Cd}^{2+}$ ions at $\mathrm{pH}=2$ (Fig. 6b). Thus, only the non-protonated form of BHIA is able to form complex with $\mathrm{Cd}^{2+}$, but at the same time it is not kept on the surface, whereas the protonated form does not interact with $\mathrm{Cd}^{2+}$. 


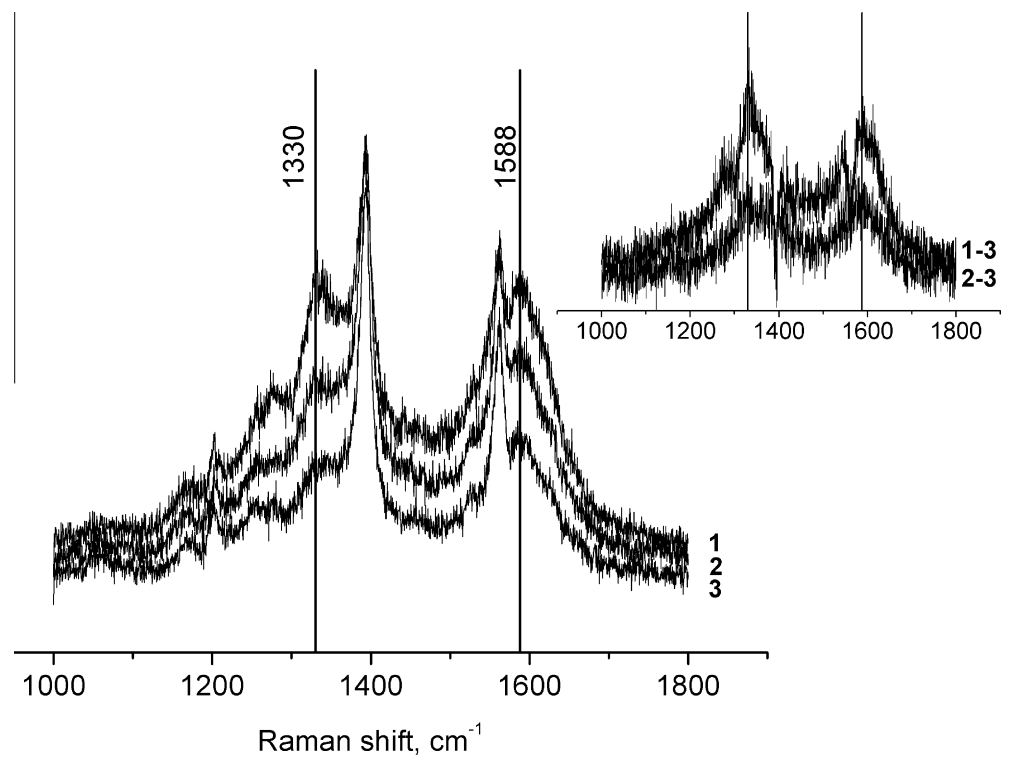

Fig. 3. SERS spectra of BHIA at stepwise changing of the $\mathrm{Cl}^{-}$anions concentration: $1-10^{-2} \mathrm{M} \mathrm{KCl}$; $2-0.1 \mathrm{M} \mathrm{KCl}$; 3-0.5 M KCl; inset: spectra after subtraction.
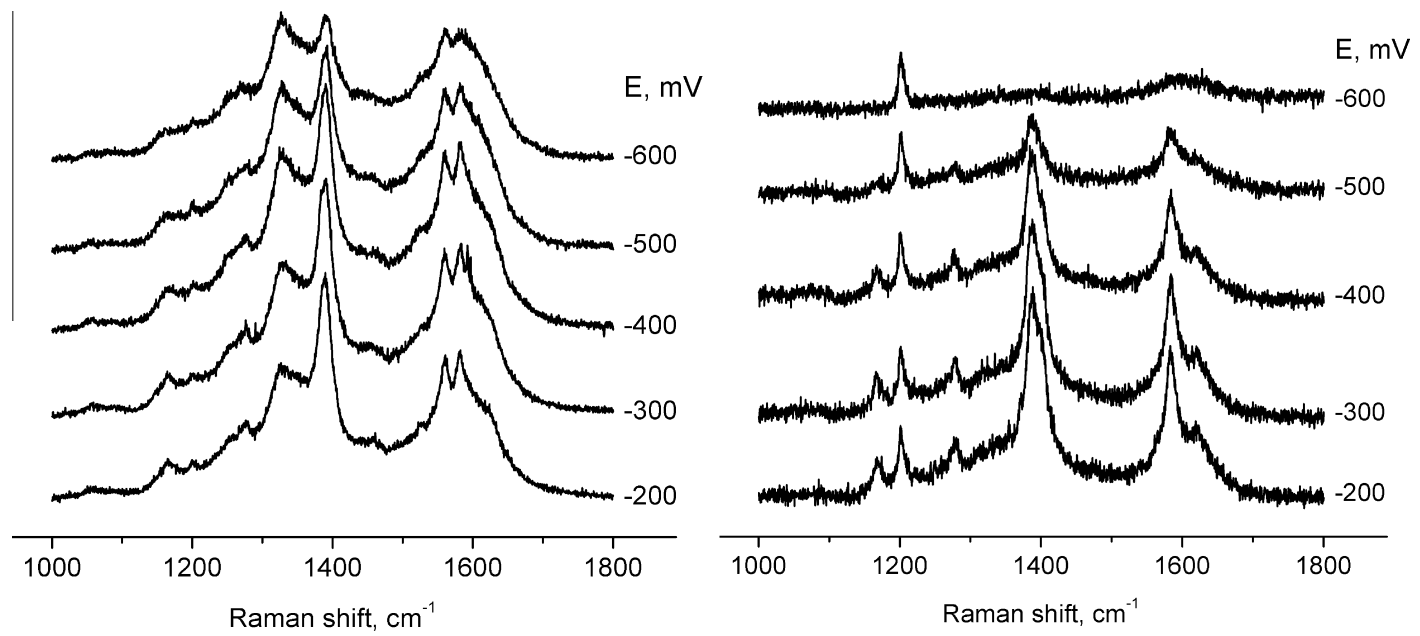

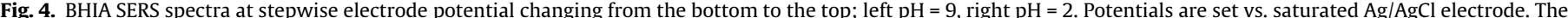
spectra are offset along the ordinate axis for better clarity.

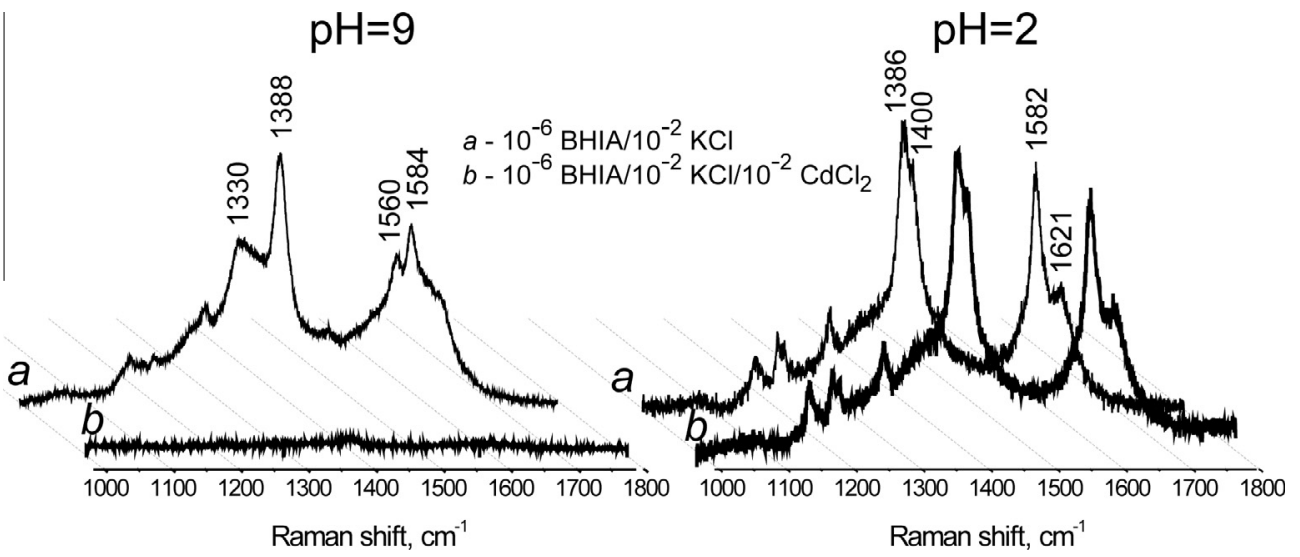

Fig. 5. BHIA SERS spectra upon $\mathrm{Cd}^{2+}$ addition to the solution at $\mathrm{pH}=9$ (left) and $\mathrm{pH}=2$ (right); a - original spectrum, $\mathrm{b}-\mathrm{spectrum}$ at $10^{-2} \mathrm{M} \mathrm{CdCl} \mathrm{L}_{2}$. 


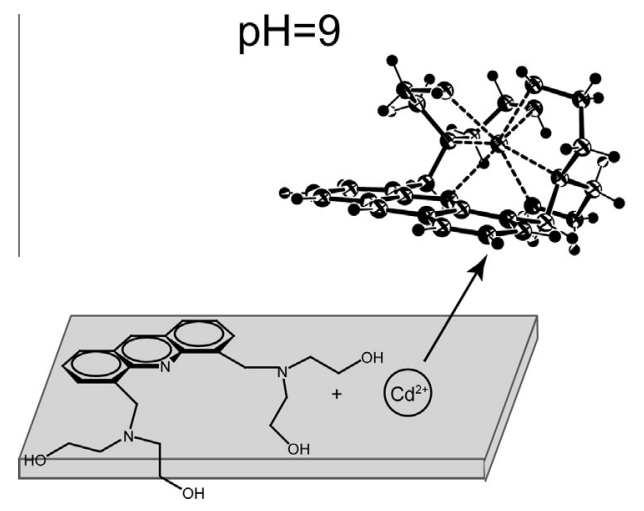

$\mathrm{pH}=2$

Fig. 6. Proposed scheme of the adsorbed BHIA interaction with $\mathrm{Cd}^{2+}$ at different $\mathrm{pH}$.

\section{Conclusions}

The SERS spectra of BHIA arise despite of the bulky substituents near of the aromatic nitrogen atom of acridine fragment when its direct interaction with a surface via the lone electron pare is unlikely. Thus, the immobilization of BHIA on active surface which gives the enhancement of optical signal is possible. The SERS spectra of BHIA undergo changes upon the variation of the $\mathrm{pH}$ of the solution caused by the protonation of the aromatic nitrogen atom. The dependence of the spectra on the electrode potential indicates that the both protonated and non-protonated forms of BHIA adsorb on silver electrode surface via the $\pi$-system of aromatic rings. Due to such adsorption mechanism the chelate fragment of molecule remains sterically available for interaction with the components of the solution. Adsorbed BHIA molecules at addition of cadmium cations in alkali solution form BHIA/Cd ${ }^{2+}$ complexes not retaining on the surface that cause the loss of SERS signal.

\section{Acknowledgements}

Authors are grateful to Galina L. Starova for the X-ray diffraction study. This work was supported by the research Grant 14-0331656 from Russian Foundation for Basic Research.

\section{References}

[1] A. Roguska, A. Kudelski, M. Pisarek, M. Lewandowska, M. Dolata, M. JanikCzachor, J. Raman Spectrosc. 40 (2009) 1652.

[2] P.R. Brejna, U. Sahaym, M.G. Norton, P.R. Griffths, J. Phys. Chem. C 115 (2011) 1444.

[3] W.B. Lacy, J.M. Williams, L.A. Wenzler, T.P. Beebe, J.M. Harris, Anal. Chem. 68 (1996) 1003.

[4] X. Wen, Y.-T. Xie, M. Wing, C. Mak, K.Y. Cheung, X.-Y. Li, R. Renneberg, S. Yang, Langmuir 22 (2006) 4836.

[5] D.H. Dressler, Y. Mastai, M. Rosenbluh, Y. Fleger, J. Mol. Struct. 935 (2009) 92.

[6] M. Muniz-Miranda, J. Raman Spectrosc. 44 (2013) 1416.

[7] M. Graff, J. Bukowska, J. Phys. Chem. B 109 (2005) 9567.

[8] G.F.S. Andrade, M.L.A. Temperini, Vibr. Spectrosc. 54 (2010) 148

[9] T.W. Lee, K. Kim, M.S. Kim, J. Mol. Struct. 274 (1992) 59.
[10] D.R.M. Godoi, Y. Chen, H. Zhu, D. Scherson, Langmuir 26 (20) (2010) 15711.

[11] M. Dendisova, L. Havranek, M. Oncak, P. Matejka, J. Phys. Chem. C 117 (2013) 21245.

[12] K.S. Shin, H.S. Lee, S.W. Joo, K. Kim, J. Phys. Chem. C 111 (2007) 15223.

[13] C. Ma, J.M. Harris, Langmuir 27 (2011) 3527.

[14] P. Cao, R. Gu, Z. Tian, J. Phys. Chem. B 107 (2003) 769.

[15] A. Jaworska, K. Malek, K.M. Marzec, M. Baranska, Spectrochim. Acta A 129 (2014) 624.

[16] Y. Chao, Q. Zhou, Y. Li, Y. Yan, Y. Wu, J. Zheng, J. Phys. Chem. C 111 (2007) 16990.

[17] N. Ohta, I. Yagi, J. Phys. Chem. C 112 (2008) 17603.

[18] S. Sarkar, M. Pradhan, A.K. Sinha, M. Basu, T. Pal, Chem. Eur. J. 18 (2012) 6335.

[19] Y. Wang, H. Wei, B. Li, W. Ren, S. Guo, S. Dong, E. Wang, Chem. Commun. 48 (2007) 5220.

[20] H.T. Temiz, I.H. Boyaci, I. Grabchev, U. Tamer, Spectrochim. Acta A 116 (2013) 339.

[21] A.R. Bizzarri, S. Cannistraro, Nanomed. NBM 3 (2007) 306.

[22] E.V. Potapkina, A.S. Denisova, L.A. Myund, A.A. Makarov, E.M. Dem'yanchuk, J. Mol. Struct. 996 (2011) 128.

[23] H. Chang, K.-C. Hwang, JACS 106 (1984) 6586.

[24] G.F.S. Andrade, M.L.A. Temperini, J. Raman Spectrosc. 40 (2009) 1989.

[25] A. Kaczor, K. Malek, M. Baranska, J. Phys. Chem. C 114 (2010) 3909.

[26] M. Muniz-Miranda, J. Phys. Chem. A 104 (2000) 7803.

[27] S. Pande, J. Chowdhury, T. Pal, J. Phys. Chem. C 115 (2011) 10497.

[28] K. Zawada, J. Bukowska, J. Mol. Struct. 555 (2000) 425.

[29] E.V. Solovyeva, L.A. Myund, E.M. Dem'yanchuk, A.A. Makarov, A.S. Denisova, J. Mol. Struct. 1034 (2013) 19.

[30] R. Brayner, R. Iglesias, S. Truong, Z. Beji, N. Felidj, F. Fievet, J. Aubard, Langmuir 26 (22) (2010) 17465.

[31] S.T. Oh, K. Kim, M.S. Kim, J. Phys. Chem. 95 (1991) 8844.

[32] S.K. Kim, D. Seo, S.J. Han, G. Son, I. Lee, C. Lee, K.D. Lee, J. Yoon, Tetrahedron 64 (2008) 6402.

[33] H.N. Lee, H.N. Kim, K.M.K. Swamy, M.S. Park, J. Kim, H. Lee, K.-H. Lee, S. Park, J Yoon, Tetrahedron Lett. 49 (2008) 1261.

[34] Y. Wang, X.Y. Hu, L. Wang, Z.B. Shang, J.B. Chao, W.J. Jin, Sens. Actuators B 156 (2011) 126.

[35] J. Kertesz, B. Bognar, A. Kormos, I. Moczar, P. Baranyai, M. Kubinyi, T. Kalai, K Hideg, P. Huszthy, Tetrahedron 67 (2011) 8860.

[36] Y. Zhou, Q. Huang, Q. Zhang, Y. Min, E. Wang, Spectrochim. Acta Part A 137 (2015) 33.

[37] E.V. Solovyeva, L.A. Myund, G.L. Starova, E.M. Dem'yanchuk, A.A. Makarov, A.S Denisova, J. Mol. Struct. 1063 (2014) 235.

[38] S.G. Schulman, A.C. Capomacchia, J. Am. Chem. Soc. 95 (1973) 2763.

[39] A. Kudelski, B. Pettinger, Chem. Phys. Lett. 321 (2000) 356.

[40] J.C. Tsang, J.E. Demuth, P.N. Sanda, J.R. Kirtley, Chem. Phys. Lett. 76 (1980) 54

[41] D.D. Kulkarni, S. Kim, A.G. Fedorov, V.V. Tsukruk, Adv. Funct. Mater. 22 (2012) 2129. 